\title{
Expression of prostate-specific membrane antigen in renal cortical tumors
}

\author{
Hikmat A Al-Ahmadie ${ }^{1}$, Semra Olgac ${ }^{2}$, Polly D Gregor ${ }^{3}$, Satish K Tickoo ${ }^{2}$, Samson W Fine ${ }^{2}$ \\ G Varuni Kondagunta ${ }^{3}$, Howard I Scher ${ }^{3}$, Michael J Morris ${ }^{3}$, Paul Russo ${ }^{4}$, Robert J Motzer ${ }^{3}$ \\ and Victor E Reuter ${ }^{2}$ \\ ${ }^{1}$ Department of Pathology, University of Chicago, Chicago, IL, USA; ${ }^{2}$ Department of Pathology, Memorial \\ Sloan-Kettering Cancer Center, New York, NY, USA; ${ }^{3}$ Department of Medicine, Memorial Sloan-Kettering \\ Cancer Center, New York, NY, USA and ${ }^{4}$ Department of Urology, Memorial Sloan-Kettering Cancer Center, \\ New York, NY, USA
}

\begin{abstract}
Prostate-specific membrane antigen is a type II membrane glycoprotein that is expressed in benign and neoplastic prostatic tissue and has been recently shown to be also expressed in the neovasculature of various solid malignant tumors including renal cell carcinoma. Renal cell carcinoma is a heterogeneous group of tumors with distinct morphologic and genetic characteristics and clinical behaviors. We performed immunohistochemical studies on formalin-fixed, paraffin-embedded archival material from $\mathbf{7 5}$ nephrectomies, using antibodies 13D6 against prostate-specific membrane antigen and CD31 against endothelial cells. The study included 30 clear cell renal cell carcinomas, and 15 of each of papillary and chromophobe renal cell carcinoma and oncocytoma. The extent and intensity of staining were assessed semiquantitatively. In all cases, immunoreactivity was detected only in the tumor-associated neovasculature and not in tumor cells. Clear cell renal cell carcinoma showed the most diffuse staining pattern, where $24 / 30$ cases or $\mathbf{8 0} \%$ had $>50 \%$ reactive vessels, followed by chromophobe renal cell carcinoma $(9 / 15 ; 60 \%)$ and oncocytoma $(5 / 15,33 \%)$. No diffuse staining was detected in any of the papillary renal cell carcinomas and only focal staining was detected in 11 cases $(11 / 15 ; 73 \%)$. Staining intensity was the strongest in clear cell renal cell carcinoma $(25 / 30 ; 83 \%)$ followed by chromophobe renal cell carcinoma $(9 / 15 ; 60 \%)$, oncocytoma $(8 / 15,53 \%)$ and papillary renal cell carcinoma $(5 / 15 ; 33 \%)$. In summary, prostate-specific membrane antigen is expressed in tumor-associated neovasculature of the majority of renal cortical tumors and is most diffusely and intensely expressed in clear cell renal cell carcinoma and least in papillary renal cell carcinoma. The differences in the expression of prostate-specific membrane antigen in renal cell carcinoma subtypes provide further evidence of the biological diversity of these tumors, and diagnostic and therapeutic applications of such expression can be expanded to include subtypes of renal cell carcinoma.
\end{abstract}

Modern Pathology (2008) 21, 727-732; doi:10.1038/modpathol.2008.42; published online 14 March 2008

Keywords: renal cell carcinoma; oncocytoma; PSMA; immunohistochemistry

Renal epithelial neoplasms constitute a heterogeneous group of tumors with distinct pathologic, molecular and clinical characteristics. Their clinical behavior ranges from entirely benign to highly malignant. In addition to differences in their morphology, these tumors also show a diverse immunophenotype. ${ }^{1,2}$

Correspondence: Dr VE Reuter, MD, Department of Pathology, Memorial Sloan-Kettering Cancer Center, 1275 York Avenue, New York, NY 10021, USA.

E-mail: reuterv@mskcc.org

Presented at the United States and Canadian Academy of Pathology (USCAP) 94th Annual Meeting, February 26-March 4, 2005, San Antonio, TX, USA.

Received 10 November 2007; revised 17 January 2008; accepted 18 January 2008; published online 14 March 2008
Prostate-specific membrane antigen (PSMA) is a type II transmembrane glycoprotein with an approximate molecular weight of $100 \mathrm{kDa}$. Its cDNA and gene have been cloned and sequenced and are located on the short arm of chromosome $11 .^{3-5}$ PSMA is an enzyme with both neurocarboxypeptidase and folate hydrolase activity.,

PSMA consists of three components: (1) a 19 amino-acid intracellular domain; (2) a 24 aminoacid transmembrane domain and (3) a 707 aminoacid extracellular domain. ${ }^{4}$ PSMA was initially identified using the monoclonal antibody (mAb) 7E11 that was raised against intact LNCaP cells ${ }^{8}$ and which recognizes a linear epitope in the intracellular domain. ${ }^{9}$ Subsequently, additional anti-PSMA monoclonal antibodies have been developed that 
bind to other epitopes present in the intracellular and extracellular domains. ${ }^{10-13}$

From the time of its initial characterization, PSMA was shown to be strongly expressed in prostatic glands, be it benign prostate secretory-acinar epithelium, prostatic intraepithelial neoplasia, or prostatic adenocarcinoma, ${ }^{4,14-16}$ and evidence suggests that PSMA expression is greatest in high-grade and hormone-insensitive prostate cancers. ${ }^{4,16-18}$

Besides the prostate, PSMA is expressed in several benign tissues including duodenum, kidney and breast. ${ }^{9,10,15,19}$ In these tissues, PSMA expression is limited to the epithelium and no expression is found in the vasculature. In contrast, PSMA was found to be expressed in the neovasculature of several solid malignant tumors including renal cell carcinoma. These studies, however, included only a small number of clear cell carcinoma as a part of a larger series consisting of a collection of varied solid tumors. ${ }^{10,12,19,20}$

PSMA-specific antibodies are currently in use for the diagnosis and staging of prostatic carcinoma with high risk for systemic disease. ${ }^{21,22}$ Additional applications include the therapy and imaging of patients with other solid tumors whose neovasculature is immunoreactive with PSMA, including renal cell carcinoma. ${ }^{23}$ As renal cell carcinoma is not a single disease but rather a heterogeneous group of tumors with distinct morphologic and genetic characteristics and clinical behaviors, imaging studies such as computed tomography and color Doppler ultrasound have been able, in select cases, to predict the histologic subtypes of renal tumors preoperatively based on the distinct architecture and vascularity of these tumors. ${ }^{24,25}$

We have conducted a study to determine whether PSMA is differentially expressed in the vessels associated with distinct types of renal epithelial neoplasms and whether it could be used as a marker of subtype.

\section{Materials and methods}

\section{Tissue Specimens}

The study included a cohort of 75 nephrectomies performed at our institution, including 30 clear cell renal cell carcinomas, 15 papillary renal cell carcinomas, 15 chromophobe renal cell carcinomas and 15 oncocytomas.

One representative section of each case was selected for analysis. The staining pattern was assessed in tumor cells, tumor-associated vessels and adjacent normal kidney. We applied a semiquantitative analysis and the score reflects the percentage of vessels staining for PSMA (or tumor cells if any) as well as the intensity of the stain.

It was considered diffuse if $>50 \%$ of the tumorassociated vessels were immunoreactive and focal if $<50 \%$ of the tumor-associated vessels were immunoreactive. Staining intensity was graded on a scale of $1-3$, where a staining intensity of 2 or 3 was considered strong.

\section{Monoclonal Antibody}

We made use of 13D6, a mouse mAb created using an immune complex containing human PSMA protein as an immunogen. ${ }^{13}$ This antibody binds to an epitope in the extracellular domain of PSMA and shows specific recognition in both fresh, frozen, ethanol-fixed and formalin-fixed paraffin-embedded tissues. This antibody was developed at our institution and is not commercially available.

To assess tumor vascularity, sequential sections were stained for the endothelial marker CD31 (Dako, Carpenteria, CA, USA).

\section{Immunohistochemistry}

Formalin-fixed, paraffin-embedded sections were cut at $4-\mu \mathrm{m}$ thickness, deparaffinized in xylene and rehydrated in graded ethanols, then transferred to $\mathrm{dH}_{2} \mathrm{O}$. Tissue sections were then pretreated by citric acid for $30 \mathrm{~min}$ at $98-99^{\circ} \mathrm{C}$, quenched in $3 \%$ $\mathrm{H}_{2} \mathrm{O}_{2}$ for $5 \mathrm{~min}$, washed with water and transferred to PBS. Sections were then placed in 1\% BSA/PBS for $1 \mathrm{~min}$ and incubated in a humidity chamber for 10 min with proper suppressor serum $11: 20$ in $2 \%$ BSA/PBS). Primary antibody was diluted with $2 \%$ BSA/PBS to an appropriate concentration of 1:1500 for 13D6, and 1:500 for CD31. 100-200 $\mu \mathrm{l}$ of diluted primary antibody was added and incubated overnight at $4^{\circ} \mathrm{C}$ in a moist chamber. Sections were thoroughly washed with PBS and biotinylated secondary antibody (horse anti-mouse IgG) was added. The slides were then thoroughly washed with PBS, and peroxide conjugated streptavidin was added to the slides (Dako, P397) at 1:500 in 1\% BSA/PBS, and incubated for $1 \mathrm{~h}$. The slides were thoroughly washed with PBS, stained in a bath of $0.06 \%$ DAB (in PBS) that had been filtered and to which $10 \mu \mathrm{l} \mathrm{H}_{2} \mathrm{O}_{2} / 100 \mathrm{ml}$ had been added. The slides were then washed in $\mathrm{dH}_{2} \mathrm{O}$, counterstained with hematoxylin, dehydrated and coverslipped.

\section{Results}

The results are summarized in Table 1.

In all tissue sections stained, only tumor-associated vessels were immunoreactive, with no labeling detected in tumor cells of any case (Figure 1).

Clear cell renal cell carcinoma showed the most diffuse staining pattern (24/30 cases; $80 \%$ ) followed by chromophobe renal cell carcinoma $(9 / 15 ; 60 \%)$ and oncocytoma $(5 / 15,33 \%)$. No diffuse staining was detected in any of the papillary renal cell carcinoma cases and only focal staining was detected in 11 cases (11/15; 73\%). The staining intensity was the strongest in clear cell renal cell carcinoma 
Table 1 Results of the immunohistochemical staining of PSMA in renal cortical tumors

\begin{tabular}{lcrlrr}
\hline & \multicolumn{2}{c}{ Staining extent } & & \multicolumn{2}{c}{ Staining intensity } \\
\cline { 2 - 3 } \cline { 5 - 6 } & Diffuse (\%) & Focal (\%) & & Strong (\%) & Weak (\%) \\
\hline CRCC $(n=30)$ & $24(80)$ & $5(17)$ & & $25(83)$ & $4(13)$ \\
PRCC $(n=15)$ & $0(0)$ & $11(73)$ & & $5(33)$ & $6(40)$ \\
ChRCC $(n=15)$ & $9(60)$ & $4(27)$ & & $9(60)$ & $4(27)$ \\
ONCO $(n=15)$ & $5(33)$ & $9(60)$ & & $8(53)$ & $6(40)$
\end{tabular}

CRCC, clear cell renal cell carcinoma; ChRCC, chromophobe renal cell carcinoma; PRCC, papillary renal cell carcinoma; PSMA, prostatespecific membrane antigen; ONCO, oncocytoma.

(25/30 cases showed strong staining intensity; 83\%) followed by chromophobe renal cell carcinoma $(9 / 15 ; 60 \%)$, oncocytoma $(8 / 15,53 \%)$, and papillary renal cell carcinoma $(5 / 15 ; 33 \%)$. There was no difference in the expression of PSMA in intratumoral vessels regardless of their sizes and calibers. Moreover, in clear cell renal cell carcinoma, there was no difference in PSMA expression between tumors of low and high nuclear grades. Vessels outside of the tumor mass (native renal vessels) showed no immunoreactivity (Figure 2).

\section{Discussion}

We report the expression of PSMA in a large cohort of renal cortical tumors, including the common subtypes of renal cell carcinoma and oncocytoma. We have used antibody 13D6 that targets an epitope in the extracellular domain of the PSMA. This antibody was developed at our institution. Currently, another antibody, 3E6, that was not commercially available at the time when we performed our study, is being marketed by Dako. We have compared these two reagents and found that the staining characteristics of $3 \mathrm{E} 6$ are similar to those we report herein (data not shown).

These renal tumors express PSMA in their neovasculature, but no expression was detected in the neoplastic cells. It is clear that PSMA staining does not correlate with the biologic behavior of tumors, as the neovasculature of the rather indolent and frankly benign tumors are immunoreactive.

From the time of its characterization, PSMA was shown to be strongly expressed in prostatic tissue, including benign acinar prostatic epithelium, prostatic intraepithelial neoplasia and prostatic adenocarcinoma. ${ }^{10,16}$ In this setting, PSMA expression is restricted to the epithelial component in an apical/luminal localization.

Outside the prostate, several studies have shown PSMA expression in the neovasculature of a variety of solid tumors including RCC. ${ }^{10,12,19,20}$ Studies that addressed the expression of PSMA in renal tumors did so in a limited number of cases and then only as part of studies that included other solid tumors.
Moreover, even in these studies, only cases of clear cell type of renal cell carcinoma were included. Silver et $a l^{19}$ observed intense PSMA staining in endothelial cells of capillary vessels in peritumoral and endotumoral areas of a number of malignancies, including 8 of 17 renal cell carcinomas. Chang et al ${ }^{10}$ found PSMA expression in the neovasculature of 11 of 11 cases of clear cell renal cell carcinoma. In another study by the same author, PSMA was found expressed in all 20 cases of metastatic clear cell renal cell carcinoma. ${ }^{20}$ Liu et $a l^{12}$ reported PSMA expression in vasculature of 9/9 renal cell carcinomas but without describing either the tumor subtype or stage.

The findings in our study are in agreement with previous reports of PSMA expression in neovasculature of clear cell renal cell carcinoma. Furthermore, we showed that PSMA is expressed in neovasculature of other renal cortical tumors including chromophobe renal cell carcinoma, oncocytoma and papillary renal cell carcinoma. This expression, however, varied in extent and intensity depending on the tumor type and was reflective of the distribution of intratumoral vessels in these cases. Clear cell renal cell carcinoma showed the most intense and most extensive expression of PSMA, whereas papillary renal cell carcinoma showed the least in both extent and intensity. As evidenced by the endothelial marker CD31, we also found that clear cell renal cell carcinoma is the most vascularized of these tumors and papillary renal cell carcinoma is the least vascularized (Figure 1).

This difference in PSMA expression may be a further evidence of the heterogeneity of renal cortical tumors, which are driven by distinct oncogenic pathways. In clear cell renal cell carcinoma, the VHL gene plays a major role in its oncogenesis that involves interaction of the VHL complex with the hypoxia-inducible factor- $1 \alpha$ (HIF1- $\alpha$ ). A mutated or hypermethylated, nonfunctional VHL complex loses its ability to target HIF1- $\alpha$ for degradation, thus leading to its accumulation. This in turn leads to upregulation of a number of downstream growth factors, including vascular endothelial growth factor, glucose transporter, platelet-derived growth factor, transforming growth factor- $\alpha$ and erythropoietin. ${ }^{26}$ These factors are believed to be involved in the development and growth of clear cell renal cell carcinoma. More importantly, understanding the VHL gene pathway has led to development of a number of molecular targeting strategies with small molecules or antibodies for potential treatment of patients with advanced clear cell renal cell carcinoma. ${ }^{26}$ Thus, the expression of PSMA in neovasculature of renal tumors may make it a potential therapeutic target for advanced stages of these tumors.

Imaging studies have attempted to predict the histological subtypes of renal tumors. The results of such studies are encouraging and to some degree echo the architectural differences of these tumors as 

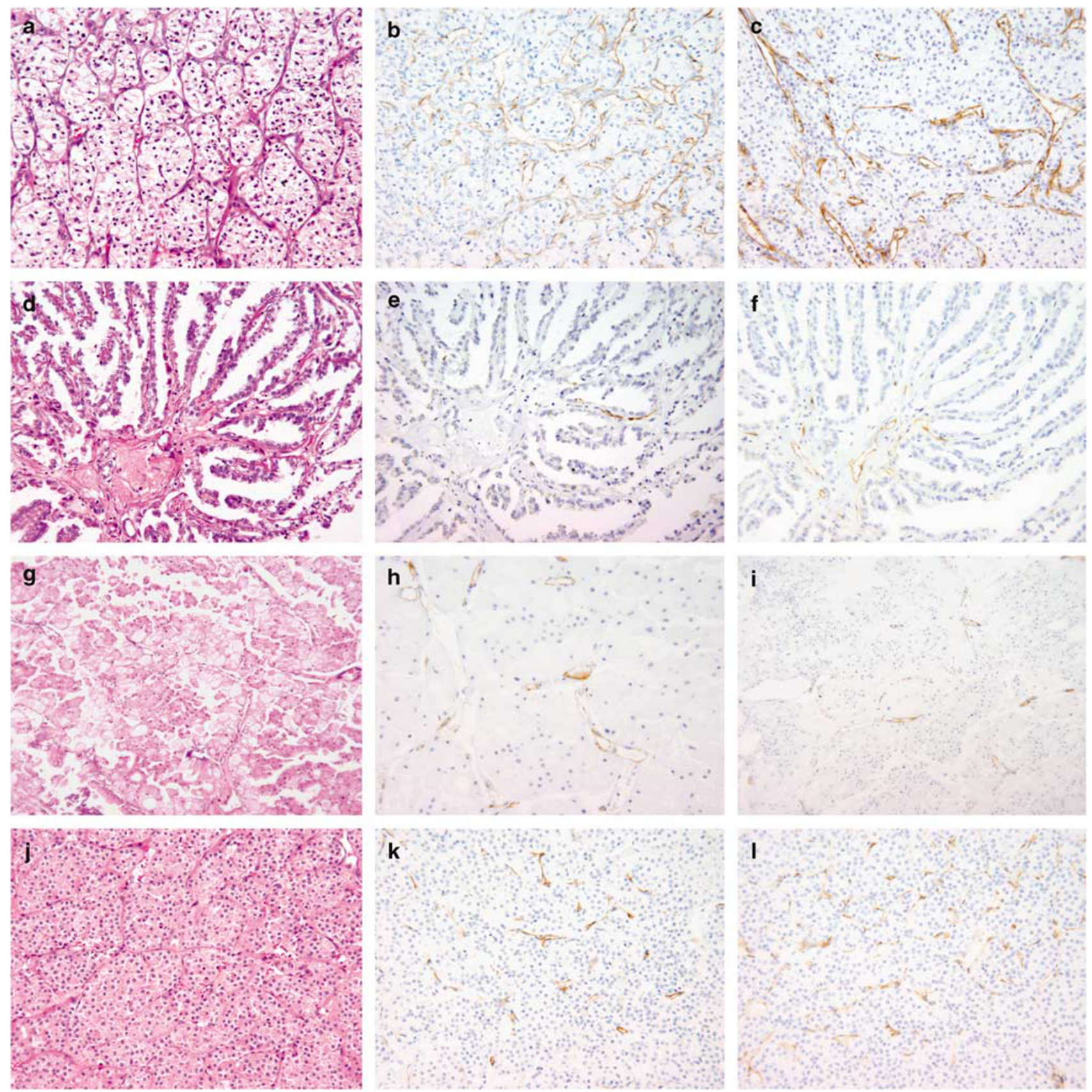

Figure 1 Differential PSMA expression in renal cortical tumors. Panels a, d, g and j were stained with H\&E. Panels b, e, h and k were stained by immunohistochemistry for 13D6. Panels c, f, i and l were stained by immunohistochemistry for CD31. In the example of clear cell renal cell carcinoma (a), there is a diffuse and intense expression of PSMA that involves nearly the entire tumor vasculature (b). Note that the expression is limited to tumor vessels with a complete absence of staining in the adjacent tumor cells. In the example of papillary renal cell carcinoma (d), very few vessels are immunoreactive and these exhibit faint intensity (e). Once again we note the lack of expression of PSMA in all tumor cells. In chromophobe renal cell carcinoma (g) and oncocytoma (j), a similar pattern of staining is present in which the vessels are the only tissue highlighted by the stain (h, k). By CD31 staining, clear cell renal cell carcinoma is clearly the most vascularized of the renal cortical tumors (c) and papillary renal cell carcinoma is the least vascularized (f). In chromophobe renal cell carcinoma and oncocytoma, CD31 highlights the variable vascularity of these tumors and shows similar, albeit slightly more diffuse and intense labeling than PSMA (i, l). Thus, PSMA expression is clearly parallel to the pattern displayed by the vascular marker CD31. This expression can now be seen as a reflection of the variable innate vascularity of these tumors rather than representing a lack of expression in uniformly vascularized tumors.

seen histologically. For example, clear cell renal cell carcinoma, some oncocytomas and some chromophobe renal cell carcinomas have increased blood flow as evidenced by color flow Doppler ultrasound, while most papillary renal cell carcinomas are hypovascular. ${ }^{24,25,27}$ These findings are consistent with the observations in this study that the vascular density and, likewise, the intensity and extent of 

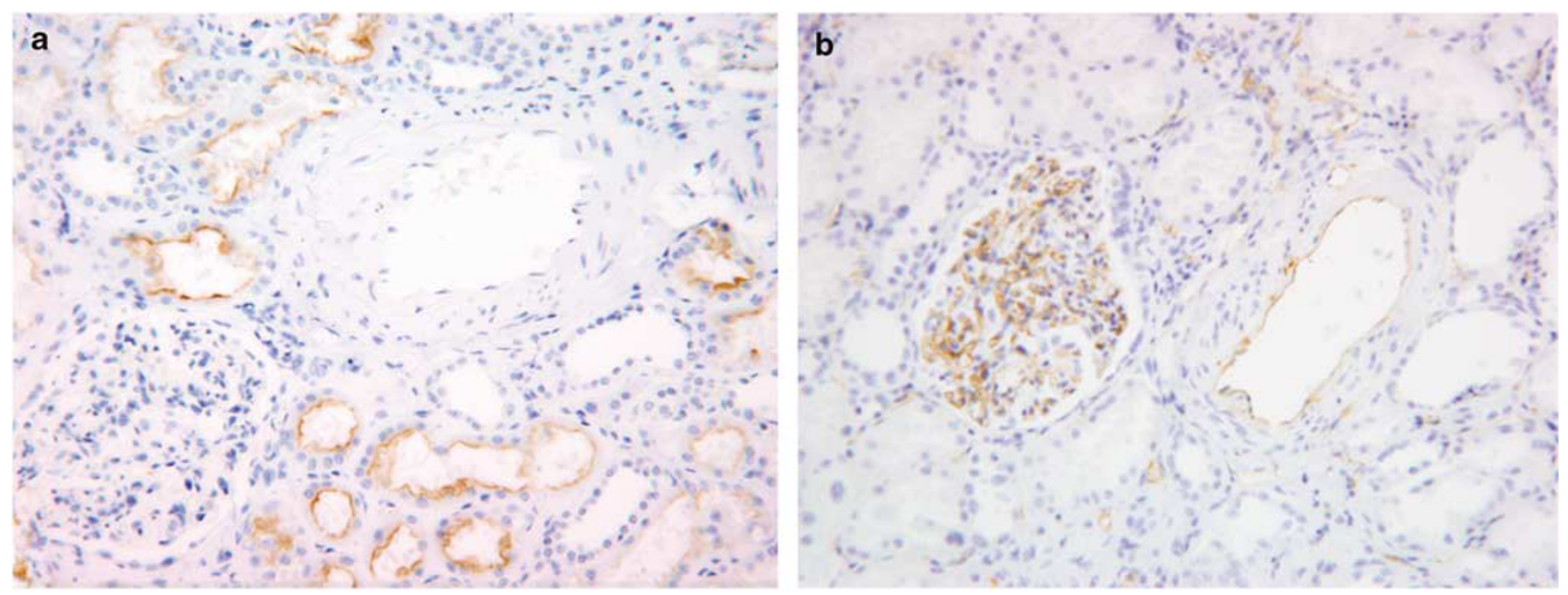

Figure 2 In non-neoplastic renal tissue adjacent to tumor, PSMA was exclusively expressed in the epithelial cells of the proximal tubules and absent in native renal vessels, glomeruli and stroma (a). In comparison, CD31 highlights the endothelial cells of vessels and glomeruli (b).

PSMA expression are the strongest in clear cell renal cell carcinoma and the weakest in papillary renal cell carcinoma.

By utilizing radiolabeled antibodies against PSMA in immunoscintigraphy scans, it has become possible to detect metastatic prostatic carcinoma ${ }^{21,22,28-30}$ and other solid tumors including renal cell carcinoma. ${ }^{23,31}$ Similarly, novel anti-PSMA mAb-based therapies have been applied to select patients with prostatic carcinoma ${ }^{30,32-35}$ and to patients with advanced stages of other solid tumors including renal cell carcinoma. ${ }^{23,36,37}$ The results of our study indicate that anti-PSMA mAb-based diagnostic and therapeutic modalities may be expanded to include antineovasculature targeting for at least some subtypes of renal cortical tumors.

The expression of PSMA in several benign tissue types may lead to potential side effects of antiPSMA therapies. However, the targets of other mAbs that are currently in clinical trials or Food and Drug Administration-approved for clinical use are also not exclusively expressed by the tumors. ${ }^{37-39}$ In one clinical trial, patients with metastatic prostatic adenocarcinoma were subjected to radiolabeled anti-PSMA mAb that accurately targeted the tumor in the majority of bone and soft tissue metastases, whereas no targeting to benign tissues and other organs was seen. ${ }^{30}$ The lack of targeting of these organs may be attributed to the fact that PSMA is expressed in luminal sites that are thought to be inaccessible to circulating antibodies. Nonetheless, caution and close monitoring should always be applied in these situations for possible side effects.

In summary, PSMA is expressed in the tumor neovasculature of renal cortical tumors, but the percentage and intensity vary among these tumor types. Tumor cells and the native vasculature lack expression in all types. The mechanism by which PSMA expression is induced in tumor neovasculature remains unknown, although the fact that it is expressed in both malignant and benign renal tumors suggests that expression is not related to biological potential.

\section{References}

1 Skinnider BF, Amin MB. An immunohistochemical approach to the differential diagnosis of renal tumors. Semin Diagn Pathol 2005;22:51-68.

2 Eble JN, Togashi K, Pisani P. Tumors of the Kidney. In: Eble JN, Sauter G, Epstein JI, Sesterhenn IA (eds). Pathology and Genetics Tumours of the Urinary System and Male Genital Organs, Vol, IARC Press: Lyon, 2004, pp 9-87.

3 O'Keefe DS, Su SL, Bacich DJ, et al. Mapping, genomic organization and promoter analysis of the human prostate-specific membrane antigen gene. Biochim Biophys Acta 1998;1443:113-127.

4 Israeli RS, Powell CT, Fair WR, et al. Molecular cloning of a complementary DNA encoding a prostate-specific membrane antigen. Cancer Res 1993;53:227-230.

5 Rinker-Schaeffer CW, Hawkins AL, Su SL, et al. Localization and physical mapping of the prostate-specific membrane antigen (PSM) gene to human chromosome 11. Genomics 1995;30:105-108.

6 Carter RE, Feldman AR, Coyle JT. Prostate-specific membrane antigen is a hydrolase with substrate and pharmacologic characteristics of a neuropeptidase. Proc Natl Acad Sci USA 1996;93:749-753.

7 Pinto JT, Suffoletto BP, Berzin TM, et al. Prostatespecific membrane antigen: a novel folate hydrolase in human prostatic carcinoma cells. Clin Cancer Res 1996;2:1445-1451.

8 Horoszewicz JS, Kawinski E, Murphy GP. Monoclonal antibodies to a new antigenic marker in epithelial prostatic cells and serum of prostatic cancer patients. Anticancer Res 1987;7:927-935.

9 Troyer JK, Beckett ML, Wright Jr GL. Detection and characterization of the prostate-specific membrane 
antigen (PSMA) in tissue extracts and body fluids. Int J Cancer 1995;62:552-558.

10 Chang SS, Reuter VE, Heston WD, et al. Five different anti-prostate-specific membrane antigen (PSMA) antibodies confirm PSMA expression in tumor-associated neovasculature. Cancer Res 1999;59:3192-3198.

11 Grauer LS, Lawler KD, Marignac JL, et al. Identification, purification, and subcellular localization of prostate-specific membrane antigen PSM' protein in the LNCaP prostatic carcinoma cell line. Cancer Res 1998;58:4787-4789.

12 Liu H, Moy P, Kim S, et al. Monoclonal antibodies to the extracellular domain of prostate-specific membrane antigen also react with tumor vascular endothelium. Cancer Res 1997;57:3629-3634.

13 Gregor PD, Wolchok JD, Turaga V, et al. Induction of autoantibodies to syngeneic prostate-specific membrane antigen by xenogeneic vaccination. Int J Cancer 2005;116:415-421.

14 Sokoloff RL, Norton KC, Gasior CL, et al. A dualmonoclonal sandwich assay for prostate-specific membrane antigen: levels in tissues, seminal fluid and urine. Prostate 2000;43:150-157.

15 Lopes AD, Davis WL, Rosenstraus MJ, et al. Immunohistochemical and pharmacokinetic characterization of the site-specific immunoconjugate CYT-356 derived from antiprostate monoclonal antibody 7E11-C5. Cancer Res 1990;50:6423-6429.

16 Wright Jr GL, Grob BM, Haley C, et al. Upregulation of prostate-specific membrane antigen after androgendeprivation therapy. Urology 1996;48:326-334.

17 Bostwick DG, Pacelli A, Blute M, et al. Prostate specific membrane antigen expression in prostatic intraepithelial neoplasia and adenocarcinoma: a study of 184 cases. Cancer 1998;82:2256-2261.

18 Kawakami M, Nakayama J. Enhanced expression of prostate-specific membrane antigen gene in prostate cancer as revealed by in situ hybridization. Cancer Res 1997;57:2321-2324.

19 Silver DA, Pellicer I, Fair WR, et al. Prostate-specific membrane antigen expression in normal and malignant human tissues. Clin Cancer Res 1997;3:81-85.

20 Chang SS, Reuter VE, Heston WD, et al. Metastatic renal cell carcinoma neovasculature expresses prostatespecific membrane antigen. Urology 2001;57:801-805.

21 Brassell SA, Rosner IL, McLeod DG. Update on magnetic resonance imaging, ProstaScint, and novel imaging in prostate cancer. Curr Opin Urol 2005;15:163-166.

22 Wong TZ, Turkington TG, Polascik TJ, et al. ProstaScint (capromab pendetide) imaging using hybrid gamma camera-CT technology. AJR Am J Roentgenol 2005;184:676-680.

23 Nanus DM, Milowsky MI, Kostakoglu L, et al. Clinical use of monoclonal antibody HuJ591 therapy: targeting prostate specific membrane antigen. J Urol 2003;170: S84-S88.

24 Helenon O, Correas JM, Balleyguier C, et al. Ultrasound of renal tumors. Eur Radiol 2001;11:1890-1901.

25 Kitamura H, Fujimoto H, Tobisu K, et al. Dynamic computed tomography and color Doppler ultrasound of renal parenchymal neoplasms: correlations with histopathological findings. Jpn J Clin Oncol 2004;34: 78-81.

26 Linehan WM, Walther MM, Zbar B. The genetic basis of cancer of the kidney. J Urol 2003;170:2163-2172.

27 Jinzaki M, Ohkuma K, Tanimoto A, et al. Small solid renal lesions: usefulness of power Doppler US. Radiology 1998;209:543-550.

28 Hinkle GH, Burgers JK, Neal CE, et al. Multicenter radioimmunoscintigraphic evaluation of patients with prostate carcinoma using indium-111 capromab pendetide. Cancer 1998;83:739-747.

29 Slovin SF. Targeting novel antigens for prostate cancer treatment: focus on prostate-specific membrane antigen. Expert Opin Ther Targets 2005;9:561-570.

30 Bander NH, Trabulsi EJ, Kostakoglu L, et al. Targeting metastatic prostate cancer with radiolabeled monoclonal antibody J591 to the extracellular domain of prostate specific membrane antigen. J Urol 2003;170: 1717-1721.

31 Michaels EK, Blend M, Quintana JC. 111Indiumcapromab pendetide unexpectedly localizes to renal cell carcinoma. J Urol 1999;161:597-598.

32 Morris MJ, Divgi CR, Pandit-Taskar N, et al. Pilot trial of unlabeled and indium-111-labeled anti-prostatespecific membrane antigen antibody J591 for castrate metastatic prostate cancer. Clin Cancer Res 2005;11: 7454-7461.

33 Murphy G, Tjoa B, Ragde H, et al. Phase I clinical trial: T-cell therapy for prostate cancer using autologous dendritic cells pulsed with HLA-A0201specific peptides from prostate-specific membrane antigen. Prostate 1996;29:371-380.

34 Murphy GP, Greene TG, Tino WT, et al. Isolation and characterization of monoclonal antibodies specific for the extracellular domain of prostate specific membrane antigen. J Urol 1998;160:2396-2401.

35 Zhang S, Zhang HS, Reuter VE, et al. Expression of potential target antigens for immunotherapy on primary and metastatic prostate cancers. Clin Cancer Res 1998;4:295-302.

36 Morris MJ, Pandit-Taskar N, Divgi CR, et al. Phase I evaluation of J591 as a vascular targeting agent in progressive solid tumors. Clin Cancer Res 2007;13: 2707-2713.

37 Gottlinger HG, Funke I, Johnson JP, et al. The epithelial cell surface antigen 17-1A, a target for antibodymediated tumor therapy: its biochemical nature, tissue distribution and recognition by different monoclonal antibodies. Int J Cancer 1986;38:47-53.

38 Pegram MD, Lipton A, Hayes DF, et al. Phase II study of receptor-enhanced chemosensitivity using recombinant humanized anti-p185HER2/neu monoclonal antibody plus cisplatin in patients with HER2/ neu-overexpressing metastatic breast cancer refractory to chemotherapy treatment. J Clin Oncol 1998;16: 2659-2671.

39 Chang SS, Heston WD. The clinical role of prostatespecific membrane antigen (PSMA). Urol Oncol 2002; 7:7-12. 